\title{
The Young Massive Star Environment
}

\author{
Stan Kurtz \\ Centro de Radioastronomía y Astrofísica, UNAM Morelia, Michoacán 58089, México \\ email: s.kurtz@astrosmo.unam.mx
}

\begin{abstract}
Molecular cloud cores-whether stellar, non-stellar, proto-stellar, or pre-protostellar - have come to be recognized as key elements in the study of star formation. For both high- and low-mass stars, these cores hold key information for the final make-up of the stellar cluster and the physical processes by which the cluster and/or the individual stars form. In the case of massive stars, the chemical effects extend even beyond the molecular core, reaching into the photo-dissociated region produced by the harder spectrum of the massive stars. We discuss the directions in which these studies are taking us, with special attention given to those aspects which are unique to high-mass star-forming regions.
\end{abstract}

Keywords. masers - ISM: clouds - ISM: molecules — stars: formation — stars: pre-mainsequence

\section{Introduction}

Massive stars (larger than about $10 \mathrm{M}_{\odot}$ ) are intrinsically interesting objects for study. Even more, they are astronomically important objects to study for a variety of reasons. During their short main-sequence lifetimes they deposit large amounts of energy and momentum into the interstellar medium (ISM). In the process, they heat - and ultimately destroy - the molecular clouds in which they formed. By doing so, they affect the rate and composition of star formation within a galaxy. Of particular interest from a chemical standpoint, the ultraviolet photon flux from massive stars creates $\mathrm{H}$ II regions and Photon Dominated Regions (PDRs), producing ions and radicals and driving photo-chemical reactions. The energy from the stars promotes endothermic reactions and evaporates grain mantles, resulting in order-of-magnitude abundance increases. In their death as supernovae, massive stars seed the ISM with heavy elements, accelerate cosmic rays which then heat molecular clouds, producing free radicals in the process, and destroy dust grains in the strong supernova shocks.

One can characterize a "typical" massive star, say of O5 spectral type, as being about $40 \mathrm{M}_{\odot}, 20 \mathrm{R}_{\odot}, 40,000 \mathrm{~K}$, and having a luminosity of around $3 \times 10^{5} \mathrm{~L}_{\odot}$ and a mainsequence lifetime of about $1.3 \times 10^{6} \mathrm{yr}$. Perhaps more crucially, one can characterize massive star formation regions as being complex. The Orion massive star forming region, for example, contains not only the massive stars (and many low-mass stars) of the Trapezium cluster, but it also hosts compact and diffuse H II regions, a PDR, molecular outflows, jets, a hot molecular core, masers, proplyds, etc. When all these phenomena (some evolving on very short timescales) are combined within a volume of a few tenths of a cubic parsec, and placed at a typical distance of $5 \mathrm{kpc}$, it becomes exceedingly difficult to disentangle all of the physical processes involved.

Moreover, the fact that massive star formation typically occurs within dense condensations of molecular clouds, with visual extinctions of order a thousand, renders these regions completely inaccessible at optical, and often near-IR, wavelengths. The most 


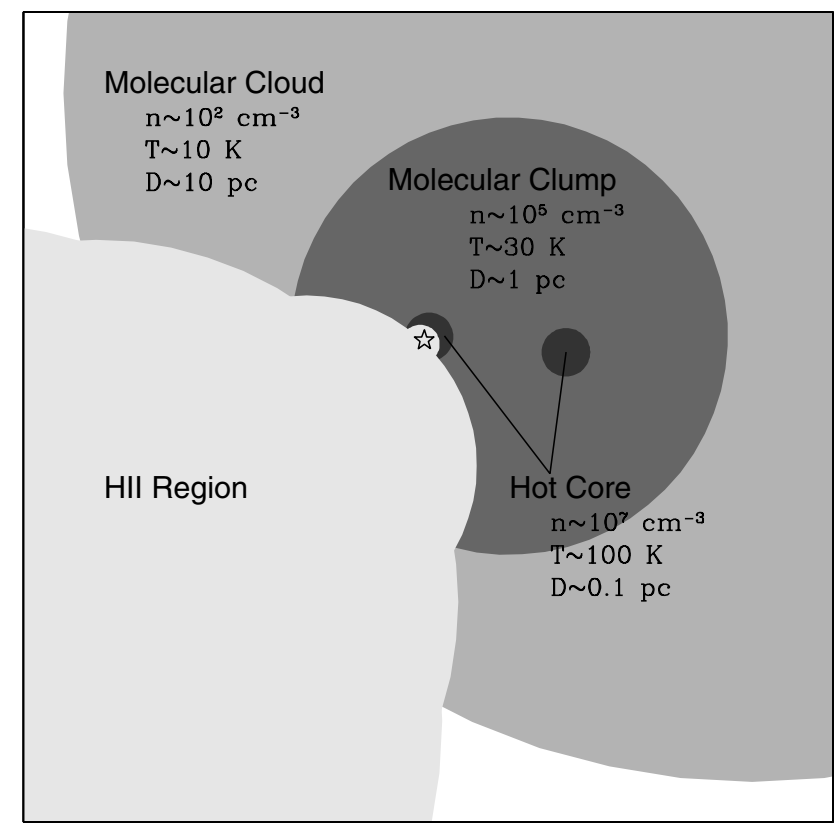

Figure 1. A schematic representation showing the hierarchy of structures within molecular clouds. Clouds have clumps, which in turn have cores. A massive star, forming within a hot molecular core, initially produces an ultracompact $\mathrm{H}$ II region, but may also give rise to much more extended ionized regions if the Lyman photon flux encounters the lower densities of the clump and the cloud. From Kim \& Koo (2001).

common tracers are seen in the radio regime: compact H II regions, masers of various chemical species, and far-IR/sub-mm continuum sources.

\section{The Star Formation Process}

Low-mass star formation studies, not hampered by the great distances, high extinctions, and complex ecologies of high-mass star formation studies, have advanced significantly. Although there are still many details to be worked out (e.g., Königl \& Pudritz 2000) and there are still controversies about the pre-protostellar stage (e.g., Vázquez et al. 2000), there is general agreement on the overall scheme of disk accretion by which low-mass stars form (e.g., Shu et al. 1987).

There is much less agreement about the process of high-mass star formation. Probably the most accepted view is essentially a scaled-up version of low-mass star formation, of infall through an accretion disk. In this view, gravitational instabilities in a molecular cloud initiate collapse of the cloud material, which proceeds on the free-fall time scale of $10^{5} \mathrm{yr}$. When sufficient material has fallen into the center of the instability, a hydrostatic core forms, which evolves on the Kelvin-Helmholtz time scale of $10^{4} \mathrm{yr}$. This is qualitatively the same as for low-mass star formation, but on shorter timescales, due to the higher densities and energies. As the molecular density continues to increase, the collapsing core eventually becomes optically thick, and the energy released by the gravitational collapse begins to heat the core, producing the so-called Hot Molecular Core phase. Eventually, nuclear reactions begin, a star is born, and the resulting ultraviolet photon flux ionizes the surrounding material, producing an ultracompact (UC) H II region. 
The various stages of this process are indicated schematically in Figure 1. The nomenclature in the literature is by no means uniform; here we adopt the usage that within the larger molecular cloud one encounters clumps of densities around $10^{5} \mathrm{~cm}^{-3}$, temperatures around $30 \mathrm{~K}$, and sizes around $1 \mathrm{pc}$. Hierarchical fragmentation gives rise to hot molecular cores within the clump, that are even denser $\left(10^{7} \mathrm{~cm}^{-3}\right)$, hotter $(>100 \mathrm{~K})$, and smaller $(0.1 \mathrm{pc})$. In this model of star formation it is the formation of the protostars within the cores that heats them, leading to the hot molecular core phase. The massive star(s), once formed, will ionize the surrounding dense molecular gas, giving rise to an UC H II region. Depending on the HMC density (possibly with gradients), and its position with respect to the clump and cloud, the UC H II region can remain ultracomapct, or can evolve into a compact H II region, with or without extended, diffuse emission (Franco et al. 2006).

As noted above, there is certainly no universal agreement on the mechanism(s) of massive star formation. A rather different proposal from the infall and accretion disk scenario is that massive stars form by the merger, or coalescence, of lower-mass stars or protostellar objects (Bonnell et al. 1998; Bally \& Zinnecker 2005). Also clear is that in some environments, different modes of massive star formation occur. An example is the "collect-and-collapse" model in which an expanding H II region sweeps up molecular material until it becomes gravitationally unstable, collapses, and forms a new massive star, near the border of the H II region. See Deharveng et al. (2005) for numerous examples. The existence of different modes of massive star formation seems likely; the existence of different mechanisms of massive star formation seems possible. Nevertheless, at present, the most popular model for the most common mode of massive star formation in the Milky Way is the scaled-up version of low-mass star formation. For the remainder of the present discussion we will presume this model.

\section{UC $\mathrm{H}$ IIs and HMCs: The Passing of the Baton}

Throughout most of the 1970s and 1980s, the chief tracers of massive star formation were the compact and ultracompact H II regions these stars formed soon after their birth. The bright radio continuum emission from these nebulae serves as an effective signpost of recent massive star formation. But naturally it was desired to push even further back toward the beginning, with some tracer of even earlier evolutionary states. In a series of papers at the beginning of the 1990s, Cesaroni, Walmsley \& Churchwell very clearly passed the baton from UC H II regions to Hot Molecular Cores as this earlier tracer (Churchwell et al. 1990; Cesaroni et al. 1991; Cesaroni et al. 1992).

Throughout the early 1990s, much of the HMC work centered on characterizing the cores: their size, temperature, density, location, and clarifying if they were internally or externally heated. Shown in Figure 2 are several examples of this work. Both the ammonia and methyl cyanide molecules have proven convenient to probe the physical properties of these regions, determine their locations, and confirm that they are, in general, internally heated. This last point is worthy of mention, because for a number of years there was some discussion about whether these objects had an internal energy source or if they were heated by an external source, typically a nearby star. Although there almost certainly are examples of molecular clumps that do not contain a massive protostar, their prospects for aiding the study of high-mass star formation were less immediate, and they were deemed of lesser interest.

Much of the characterization of HMC properties was done by people whose primary interest was the star formation process. Thankfully, other people, with more chemical interests, were also involved, and numerous studies of the chemical processes within HMC have been made, including Charnley (1997), Doty \& Neufeld (1997), Millar et al. (1997), 

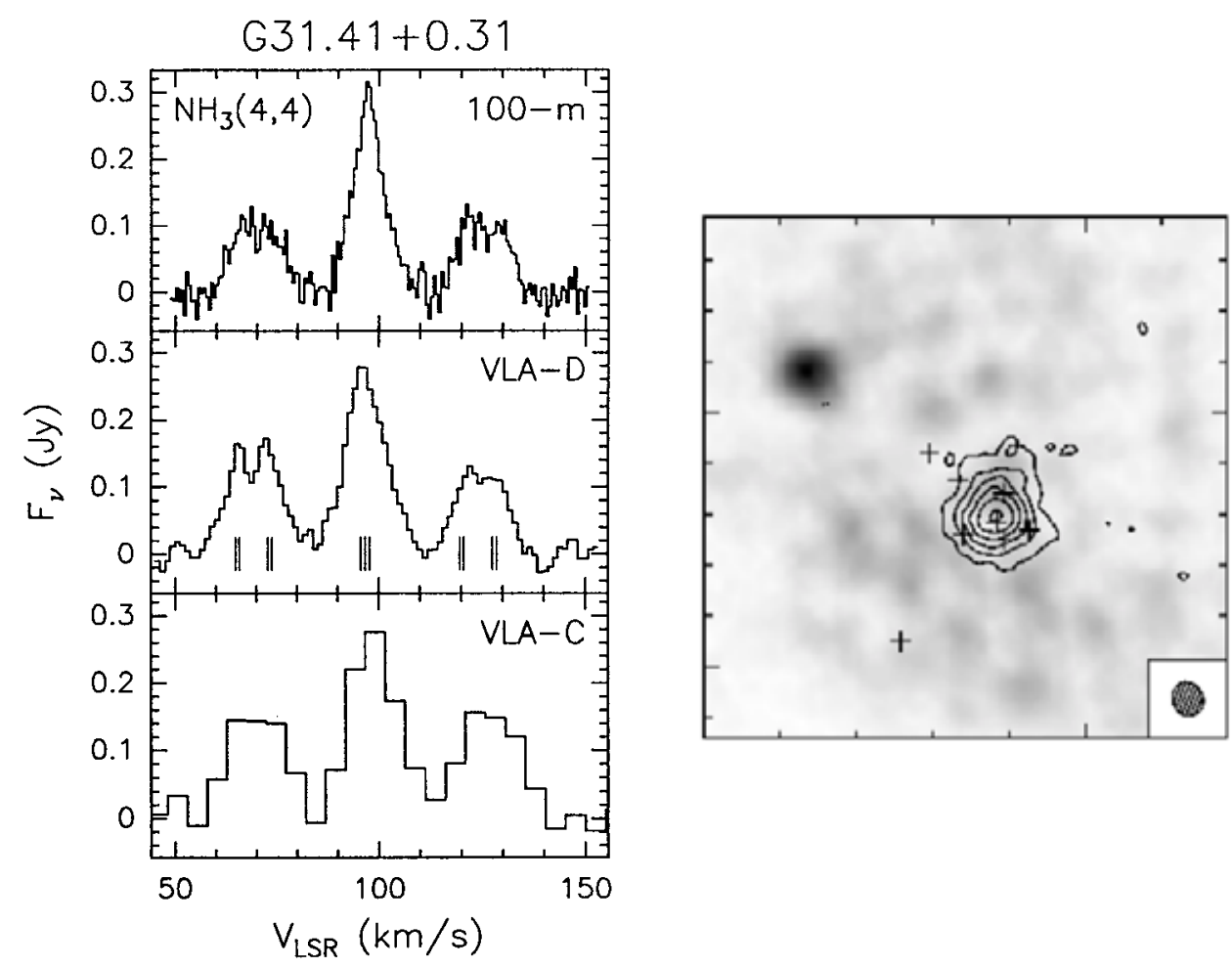

Figure 2. Left: The ammonia spectra of the G31.41+0.31 hot molecular core. The fact that we see the $(4,4)$ transition indicates the gas is hot. The fact that we see the hyperfine satellite components indicates that the gas is dense. The fact that the gas is dense even at the high angular resolution of the VLA C-configuration indicates that the gas is compact. Right: An image of the G31.41+0.31 field. The greyscale indicates ionized gas (an UC H II region is seen to the northeast) while the contours show the ammonia $(4,4)$ emission of the hot molecular core. Crosses show water masers. From Cesaroni et al. 1998.

Hatchell et al. (1998), Viti \& Williams (1999), and van Dishoeck \& Blake (1998, and references therein) to mention just a few.

In fact, chemical studies of these regions have now reached the point where they can serve as diagnostics of the star formation process. For example, studies by Viti et al. (2001) and Hatchell \& Viti (2002) show that the NS/CS abundance ratio can be used to test for the passage of C-shocks within HMC, which in turn can be used to place restrictions on the lifetime of this phase. Similarly, CS and $\mathrm{N}_{2} \mathrm{H}^{+}$observations of Zinchenko et al. (2005) coupled with models of Lintott et al. (2005) suggest an accelerated collapse rate within HMC, providing dynamically important information about the star formation process. Further information about such studies can be found in this volume, in the chapters by S. Doty and S. Viti.

\section{HMCs and HMSCs: Another Pass of the Baton}

Just as it was worthwhile to pass from UC H II regions to HMC as the earliest tracer of massive star formation, it is natural to seek an even early stage than HMC. If we ask "what comes before a hot molecular core?" presumably the answer is a warm molecular core. And presumably before a warm core, one has a cold core. The core presumably is cold because there is no internal energy source, so one might also refer to these cold cores 


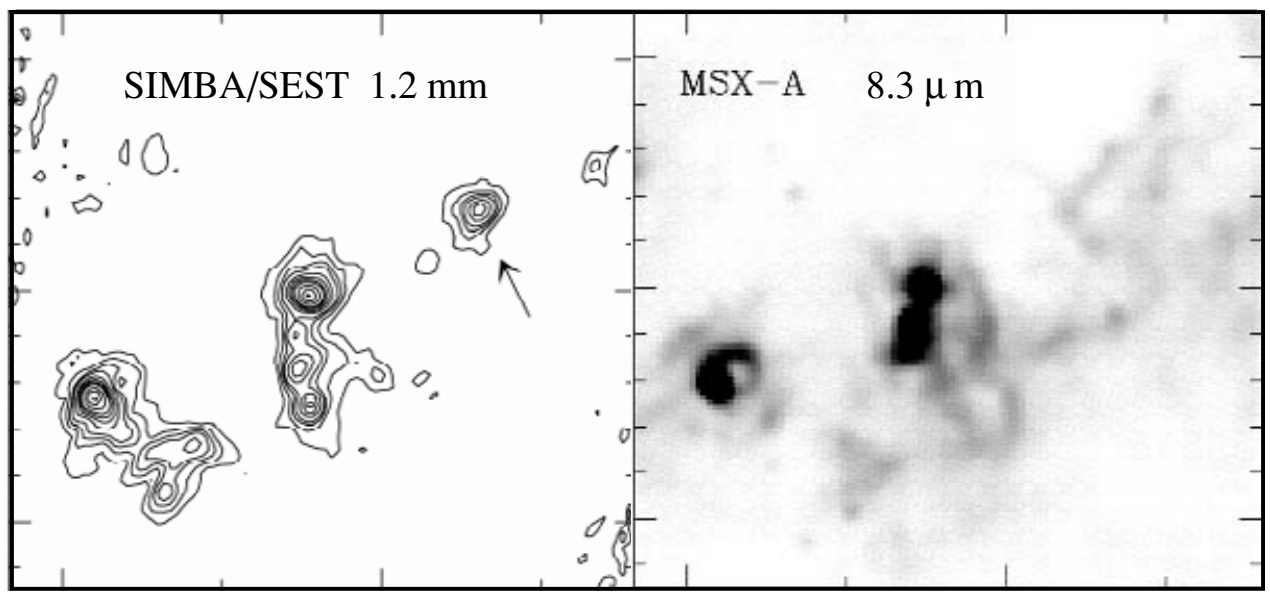

Figure 3. Left: The $1.2 \mathrm{~mm}$ continuum emission from IRAS 13080-6229. Right: The MSX A-band $(8.3 \mu \mathrm{m})$ emission from the same sky area. Apart from the diffuse $\mathrm{PAH}$ emission seen in the MSX image, the most striking difference is appearance of the northwestern source in the SEST image (indicated by the arrow). To be seen at $1.2 \mathrm{~mm}$ and absent in all MSX and IRAS bands indicates a very cold source. Images from Garay et al. (2004).

as high-mass starless cores (HMSC). Not only would this take us closer to the beginning stages, which are least understood, but hopefully it would provide a means of distinguishing between different mechanisms of star formation (for example, disk accretion versus coalescence) and - something of a holy grail - it might show us if and how the physical conditions within the cloud core determine the initial mass function of the stellar cluster that ultimately will form.

Perhaps the most cogent prediction of this earlier phase was made by Evans et al. (2002) at the Boulder Hot Star Workshop. That meeting was infamous for generating a frightful array of abbreviations, and the reference in question was no exception, introducing PreProtostellar Cores (PPCs) and Pre-Proto-cluster Cores (PPclCs). A casual perusal of this section should convince the reader that the nomenclature has, if anything, degenerated even further. Evans et al. (2002) developed a model flux density distribution for these massive $\left(4600 \mathrm{M}_{\odot}\right)$, cold $(10 \mathrm{~K})$ putative PPclCs, which, not surprisingly for so cold an object, peaks in the sub-mm. More crucial, perhaps, is that in their model the flux density falls by $2-3$ orders of magnitude going into the IRAS bands (100 to $12 \mu \mathrm{m}$ ).

So the stage was set for the search and discovery of these objects. All-sky infrared surveys, combined with pointed millimeter or sub-millimeter observations might detect these objects: something this cold should appear in the radio, but not in the infrared. A number of groups picked up the gauntlet, and they have succeeded in finding a substantial number of candidate sources.

Garay et al. (2004) report the detection of four molecular cores in the $1.2 \mathrm{~mm}$ continuum, which do not have IRAS or MSX counterparts. They derive sizes of $0.2-0.3$ pc, densities around $2 \times 10^{5} \mathrm{~cm}^{-3}$, and total masses from 400 to $2,000 \mathrm{M}_{\odot}$. The lack of emission at the IRAS and MSX (4 to $26 \mu \mathrm{m}$ ) bands implies a $17 \mathrm{~K}$ upper limit to the dust temperature within the cores. They confirm the presence of dense molecular gas with $\mathrm{CS}(2-1)$ observations (Figure 4) which shows highly supersonic linewidths of $\Delta \mathrm{V}$ $\sim 4 \mathrm{~km} \mathrm{~s}^{-1}$. If these linewidths arise from turbulence, rather than bulk motions in the gas, it may have important consequences for these massive cold cores. In particular, the contribution of turbulent pressure would allow hydrostatic equilibrium to occur at much 


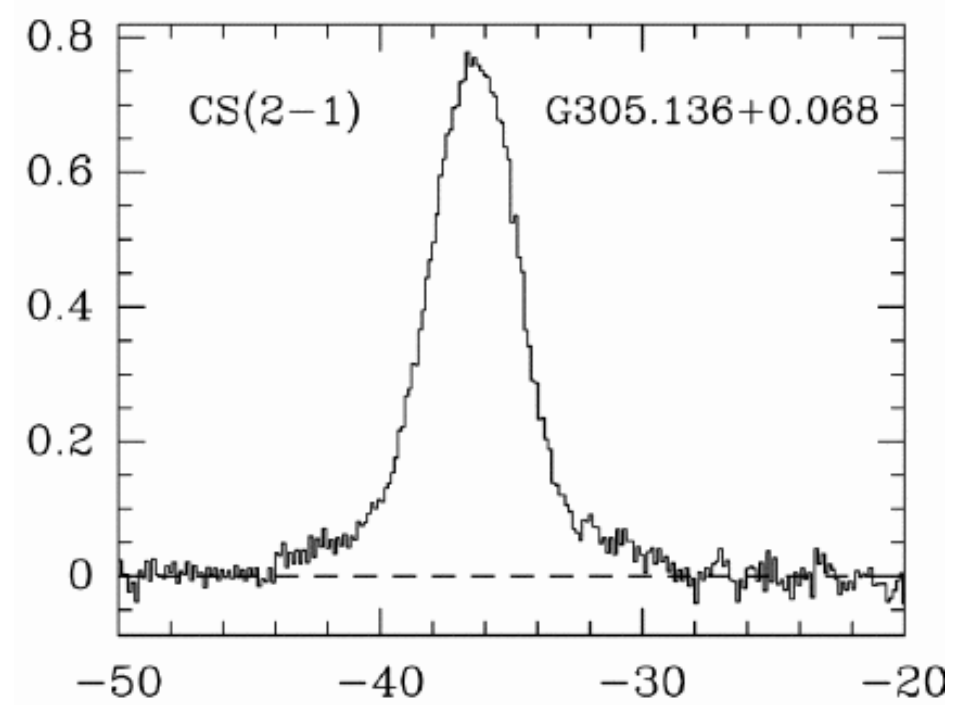

Figure 4. The CS(2-1) spectrum obtained from the IRAS 13080-6229 field (also identified as G305.136+0.069). The vertical axis is antenna temperature in Kelvins; the horizontal axis is LSR velocity in kilometers per second. Spectrum from Garay et al. (2004).

higher densities than in low-mass starless cores, which could have important implications for their chemistry.

Faúndez et al. (2004) report many additional sources from the same Garay et al. (2004) study, with typical temperatures of $32 \mathrm{~K}$. These sources may be more representative of warm cores, but they report ten sources with dust temperatures of $25 \mathrm{~K}$ or less which may prove to be cold cores. An additional candidate was reported by Forbrich et al. (2004). Egan et al. (1998), Molinari et al. (2002), Williams et al. (2004), and Ao et al. (2004) have probably observed cold and warm cores as well, although additional observations are needed to confirm this.

Several large-scale surveys of high-mass starless cores are underway. The first is SCAMPS (SCubA Massive Precluster Survey) being carried out by M. Thompson, F. Wyrowski, and collaborators. This survey, which combines JCMT observations with data from MSX and IRAS, has identified about 25 high-mass starless cores. Preliminary results indicate that core temperatures are $<35 \mathrm{~K}$, densities are of order $10^{5}-10^{6} \mathrm{~cm}^{-3}$, and masses are from 1,000-4,000 $\mathrm{M}_{\odot}$ (Thompson et al. 2006).

Klein et al. (2005) have made an extensive, multi-line survey based on a sample of 47 FIR-bright objects. Using a combination of near- and mid-IR data, together with radio observations, they have identified 12 pre-protocluster core candidates, with densities of order $10^{4} \mathrm{~cm}^{-3}$ and masses of order $10^{3} \mathrm{M}_{\odot}$. Their full sample includes objects in a range of evolutionary stages, and they develop a tentative sequence for a 5 -stage star formation process, ranging from an initially static massive cloud core up to the dispersal of the parental cloud by the newly formed cluster. Their initial, single-dish results are being followed-up by high angular resolution interferometric observations (see the discussion following the bibliography).

Sridharan et al. (2005) have examined a sample of 69 candidate high-mass protostellar objects (HMPO), comparing the $1.2 \mathrm{~mm}$ continuum with $8.3 \mu \mathrm{m}$ MSX data, and have identified 56 candidate high-mass starless cores (HMSC). Ammonia observations of these sources suggest that there are distinct populations: the starless cores candidates are 

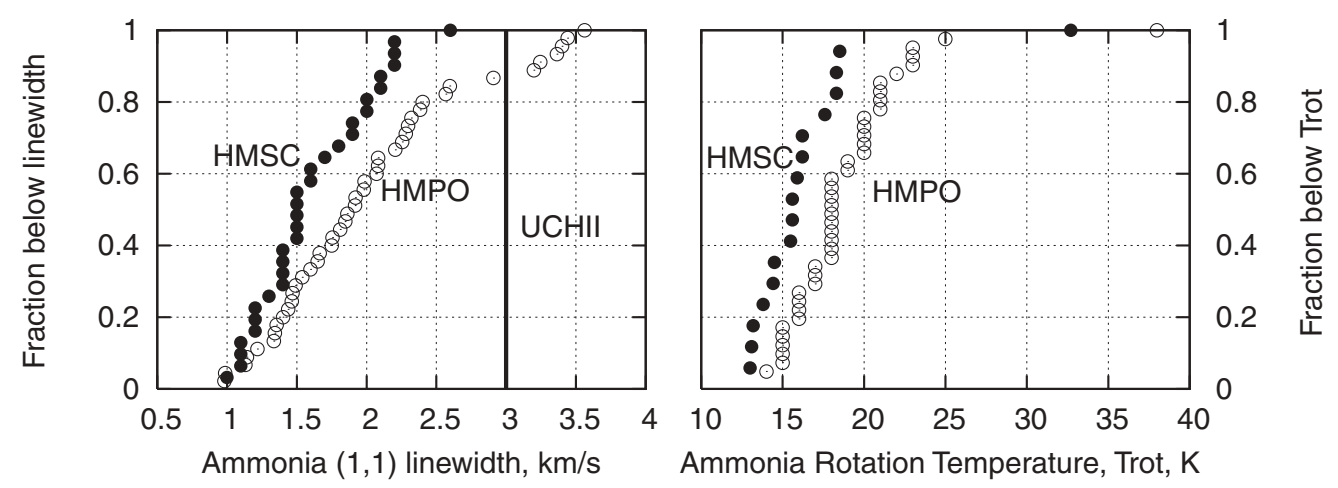

Figure 5. Cumulative probability plots (taken from Sridharan et al. 2005) show that the sample of high-mass starless core candidates is more quiescent (left) and cooler (right) than the sample of high-mass protostellar object candidates.

systematically more quiescent and colder than the protostellar objects (see Figure 5). Presumably (but not yet confirmed) the HMPOs are warmer and more turbulent because they already have a central heating source, and are more indicative of warm cores, rather than cold cores.

The above studies are all biased in the sense that they searched for cold cores in the vicinity of known or suspected massive star formation regions. Naturally it is desirable to have an un-biased search, and this is precisely the goal of the CHaMP survey (Census of High- and Medium-mass Protostars) presented at this conference by Barnes et al. (2006). Using the NANTEN telescope, they have identified $\sim 140$ molecular cores from a $60 \mathrm{deg}^{2}$ area of the galactic plane. Follow-up observations in a variety of molecular tracers are currently underway with the Mopra and ATCA telescopes. The CHaMP survey should identify a complete population of cloud cores, which will provide constraints on timescales for the various evolutionary states, among other important results.

\section{Where Do We Go from Here?}

Perhaps one of the more urgent tasks awaiting us is to confirm that in fact these are starless cores. It is conceivable, for example, that low-mass stars are merrily forming away within these cores and are simply unable to heat or otherwise disturb the core sufficiently for us to have noticed. Sensitive far- and mid-IR observations are needed to confirm this.

Assuming this is confirmed, then the studies mentioned above will present a substantial sample of cold cores. But given this sample, how will we study it? What molecular tracers will we use? The workhorse molecule of HMC, ammonia, will be relatively useless for such cores: only the $(1,1)$ inversion line is expected to be excited (see Figure 6). An alternative might be formaldehyde, which does have a significant number of transitions in the $<20 \mathrm{~K}$ range. At molecular hydrogen densities below about $10^{5} \mathrm{~cm}^{-3}$ it is super-cooled and seen in absorption (Townes \& Cheung 1969). At densities above $10^{5} \mathrm{~cm}^{-3}$, however, the collisional process that populates the lower levels is quenched, and the lines can appear in emission (Garrison et al. 1975). Whether formaldehyde will actually be useful may depend on other factors, however. For example, Young et al. (2004) have shown that in some low-mass star forming regions there is substantial depletion of formaldehyde. Such depletion, or some other as yet unrecognized chemical process, could severely restrict the usefulness of otherwise valuable tracers such as formaldehyde. 


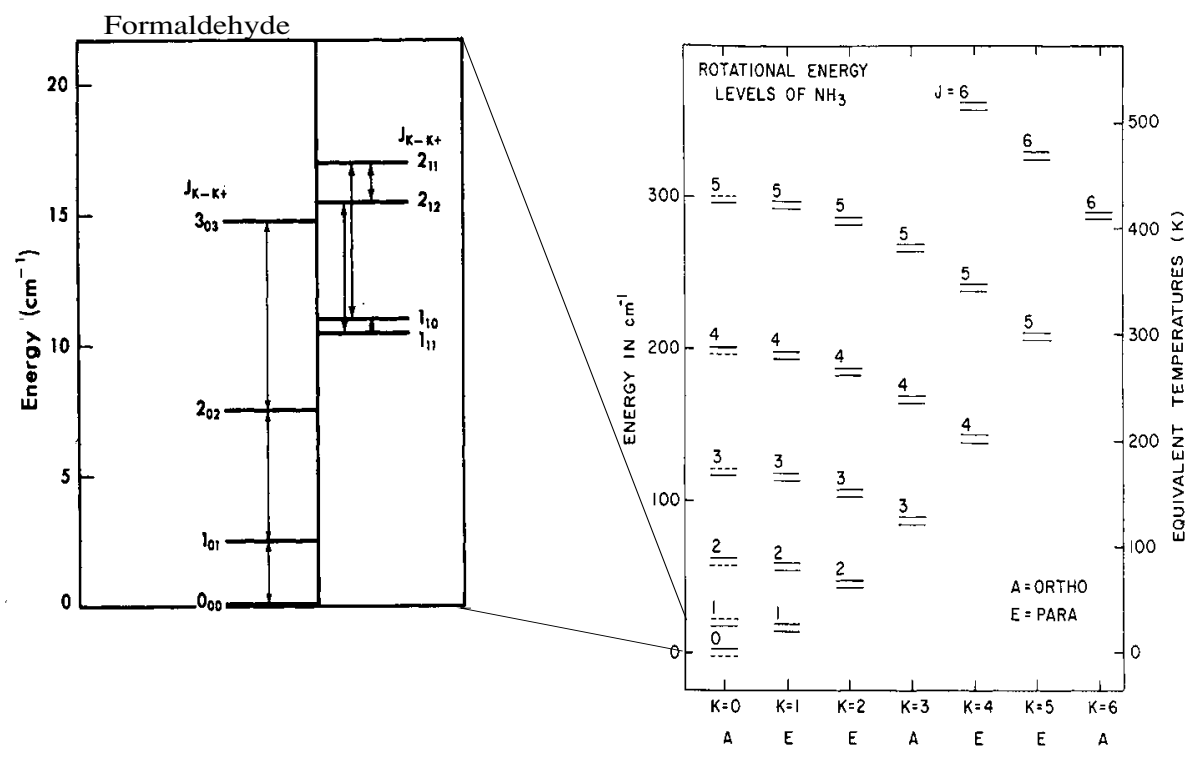

Figure 6. Level diagrams for formaldehyde and ammonia (from Townes \& Cheung 1969 and Ho \& Townes 1983, respectively). The ammonia $(1,1)$ transition is at $23 \mathrm{~K}$, the $(2,2)$ at $65 \mathrm{~K}$, and the $(3,3)$ at $125 \mathrm{~K}$; of these, only the $(1,1)$ transition at $23 \mathrm{~K}$ will be useful to probe cold cores. Formaldehyde, however, has numerous transitions at even lower excitation levels.

One of the first tasks awaiting us is the identification and verification of useful cold core tracers. Once identified, we can begin a similar process of characterizing cold cores as was done previously for hot cores. And, ultimately, we can use the knowledge gained to further our understanding of the massive star formation process.

\section{Acknowledgements}

I am grateful to Th. Henning and P. Barnes for helpful comments, and to the many conference attendees with whom I had helpful, enlightening, and very enjoyable conversations.

\section{References}

Ao, Y., Yang, J., \& Sunada, K. 2004 A. J. 128, 1716

Bally, J. \& Zinnecker, H. 2005 A. J. 129, 2281

Barnes, P. J., Yonekura, Y., Miller, A., Agars, L., Mühlegger, M., Wong, T., Ladd, E. F., Mizuno, N., \& Fukui, Y. 2006 IAU 231, (poster)

Bonnell, I. A., Bate, M. R., \& Zinnecker, H. 1998 MNRAS 298, 93

Churchwell, E., Walmsley, C. M., \& Cesaroni, R. 1990 A $\&$ A Suppl. 83, 119

Cesaroni, R., Hofner, P., Walmsley, C. M., \& Churchwell, E. 1998 A\&A 331, 709

Cesaroni, R., Walmsley, C. M., Koempe, C., \& Churchwell, E. 1991 A\& A 252, 278

Cesaroni, R., Walmsley, C. M., \& Churchwell, E. 1992 A\&A 256, 618

Charnley, S. B. 1997 Ap. J. 481, 396

Deharveng, L., Zavagno, A., \& Caplan, J. 2005 A $\& A$ 433, 565

Doty, S. \& Neufeld, D. 1997 Ap. J. 489, 122

Egan, M. P., Shipman, R. F., Price, S. D., Carey, S. J., Clark, F. O., \& Cohen, M. 1998 Ap. J. 494, L199

Evans, N. J., Shirley, Y., Mueller, K. E., \& Knez, C. 2002 ASP Conf. Ser. 267, 17

Faúndez, S., Bronfman, L., Garay, G., Chini, R., Nyman, L.-Å, \& May, J. 2004, A $\& A$ 426, 97

Forbrich, J., Scheyer, K., Posselt, B., Klein, R. \& Henning, Th. 2004, Ap. J. 602, 843 
Franco, J., García-Segura, G., \& Kurtz, S. 2006 Ap. J. submitted

Garay, G., Faúndez, S., Mardones, D., Bronfman, L., Chini, R. \& Nyman, L.-̊ 2004, Ap. J. 610,313

Garrison, B. J., Lester, W. A., Miller, W. H., \& Green, S. 1975, Ap. J. 200, L175

Hatchell, J., Millar, T. J., \& Rodgers, S. D. 1998 A $\& A$ 332, 695

Hatchell, J. \& Viti, S. 2002, A\&A 381, L33

Ho, P. T. P. \& Townes, C. H. 1983, Ann. Rev. Astron. Astroph. 21, 239

Kim, K.-T. \& Koo, B.-C. 2001, Ap. J. 549, 979

Klein, R., Posselt, B., Schreyer, K., Frobich, J., \& Henning, Th. 2005, Ap. J.S 161, in press

Königl, A., \& Pudritz, R. 2000 Protostars 83 Planets IV Eds. V. Mannings, A. Boss, \& S. Russell (Tucson: Univ. of Arizona Press), p. 759

Lintott, C. J., Viti, S., Rawlings, J. M. C., Williams, D. A., Hartquist, T. W., Caselli, P., Zinchenko, I., \& Myers, P. 2005, Ap. J. 620, 795

Millar, T. J., MacDonald, G. H., \& Gibb, A. G. 1997 A $\& A$ 325, 1163

Molinari, S., Testi, L., Rodríguez, L. F., \& Zhang, Q. 2002 Ap. J. 570, 758

Shu, F., Adams, F., \& Lizano, S. 1987 Ann. Rev. A\&A 25, 23

Sridharan, T. K., Beuther, H., Saito, M., Wyrowski, F. \& Schilke, P. 2005, Ap. J. submitted, and astro-ph/0508421

Thompson, M., Pestalozzi, M., Gibb, A., Hatchell, J., \& Wyrowski, F. 2006 IAU Symposium 227, in press

Townes, C. H. \& Cheung, A. C. 1969, Ap. J. 157, L103

van Dishoeck, E. \& Blake, G. 1998 ARAA 36, 317

Vázquez-Semadeni, E., Ostriker, E., Passot, T., Gammie, C., \& Stone, J. 2000 Protostars \& Planets IV Eds. V. Mannings, A. Boss, \& S. Russell (Tucson: Univ. of Arizona Press), p. 3

Viti, S., Caselli, P., Hartquist, T. W., \& Williams, D. A. 2001, A\&\&A 370, 1017

Viti, S. \& Williams, D. A. 1999, MNRAS 305, 755

Williams, S. J., Fuller, G. A., \& Sridharan, T. K. 2004 A\& A 417, 115

Young, K. E., Lee, J.-E., Evans, N. J., Goldsmith, P. F., \& Doty, S. D. 2004, Ap. J. 614, 252

Zinchenko, I. et al. 2005, Ap. J., submitted

\section{Discussion}

STRELNITSKI:Is it possible that the "highly supersonic" line width you mentioned $\left(\Delta v \simeq 4 \mathrm{~km} \mathrm{~s}^{-1}\right)$ is due to rotation of the core rather than to turbulence?

KURTZ: The vast majority of these sources have not yet been observed with sufficient angular resolution to address that issue. Molecular line profiles (single-dish, single-pointing) do show broad wings in some cases, suggesting bulk motion of the gas. This issue must be addressed by future observations; see the following comment by Thomas Henning.

HENNING: In our survey (Klein et al. 2005) we found a number of good candidates for high-mass protostellar cores (millimeter detection, no MIR counterparts). We found that at least one of these cores shows molecules that trace outflow activity.

KuRTZ: Thanks for that comment, Thomas. 


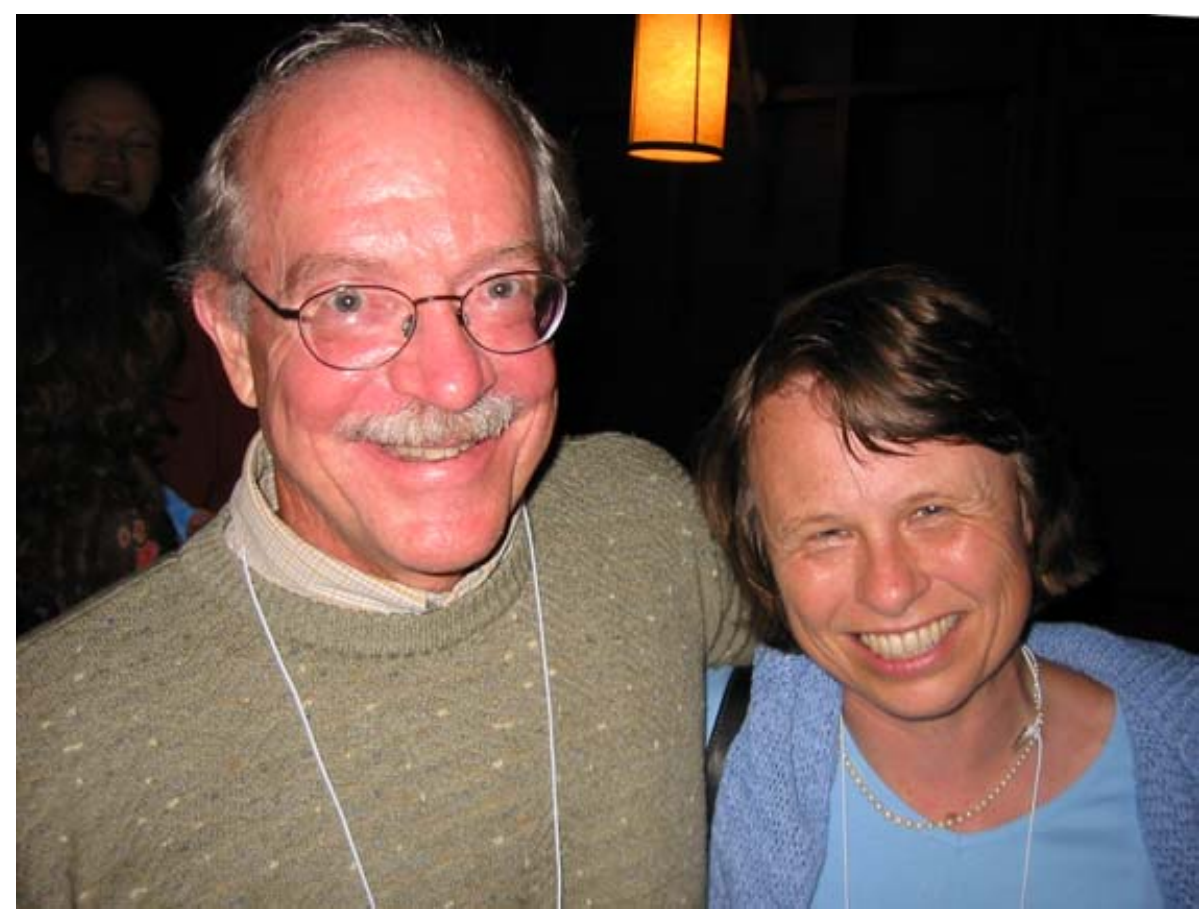

Photo: T. Snow 